\title{
A New Twist on Exciton Lifetimes
}

\author{
Varying the twist angle between layers of a 2D semiconductor can prolong \\ the duration of resonances that give the material its unique \\ optoelectronic properties.
}

\author{
By Rachel Berkowitz
}

$\square$ tructures made by stacking two semiconductor monolayers have unique optical and electronic properties, making them promising platforms for nanoelectronic and photonic devices. Central to these properties are excitons-electron-hole pairs that exist briefly between when a semiconductor absorbs a photon and when it re-emits it. As excitons in 2D semiconductors are notoriously short-lived, prolonging them is critical to optimizing the performance of optoelectronic devices based on these layered materials. Junho Choi of the University of Texas Austin and Matthias Florian of the University of Bremen, Germany, and colleagues now show that such an extension of exciton lifetimes can be achieved by varying the angle between the semiconductor layers [1].

When two semiconductor crystal sheets are stacked, their atomic lattices become superimposed to form a more complex pattern called a moiré superlattice. A rotation of one sheet
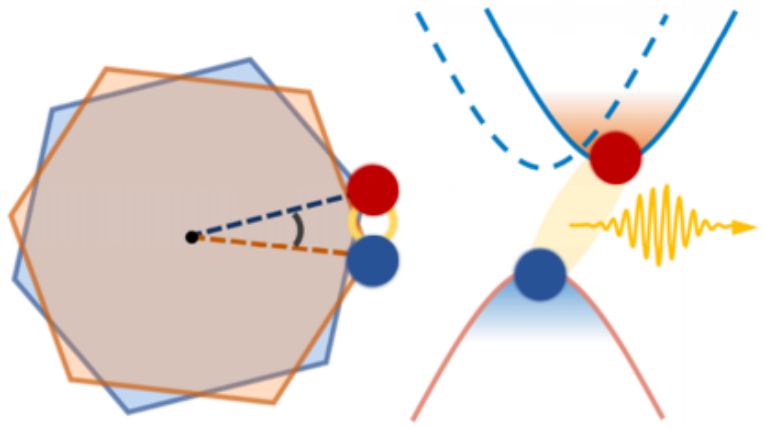

relative to the other alters that pattern and influences the bilayer's electronic properties, such as how its electrons move and how electron-hole pairs recombine. Choi, Florian, and colleagues used photoluminescence spectroscopy to investigate how this twist angle influences the dynamics of excitons whose electrons and holes reside in opposite layers. Such interlayer excitons are especially sensitive to rotations of the semiconductor sheets, so they are key to tuning the structure's charge-transport properties.

They found that a small increase in the twist angle-from 1 to 3.5 degrees-produced an order-of-magnitude increase in exciton lifetimes. Based on calculations, the researchers explained the result with a twofold mechanism involving shifts in the electrons' momentum and changes in the energy potential between the layers. These findings could provide a new approach for developing more efficient light-emitting diodes, regular arrays of quantum emitters, and other novel optoelectronic devices.

Rachel Berkowitz is a Corresponding Editor for Physics based in Vancouver, Canada.

\section{REFERENCES}

1. J. Choi et al., "Twist angle-dependent interlayer exciton lifetimes in van der Waals heterostructures," Phys. Rev. Lett. 126, 047401 (2021).

Credit: J. Choi et al. [1] 